\title{
Folkbiology: The Importance of How People Understand Nature
}

\author{
The Native Mind and the Cultural Construction of Nature, by Scott Atran and Douglas \\ Medin. Cambridge, Massachussets: The MIT Press, 2008. Pp. viii $+333, \$ 40.00$ (H/b)
}

\section{Emanuele Serrelli}

Published online: 15 July 2010

(C) Springer Science+Business Media, LLC 2010

Keywords Folkbiology · Folktaxonomy · Folkecology Biology education · Environment · Anthropology .

Cognitive psychology

Although this is a long review, I only cover perhaps two thirds of the contents of The Native Mind and the Cultural Construction of Nature, and very briefly indeed. As the press release informs us, this book draws on two decades of research and contains a huge yield of data, analyses, research protocols, and theoretical background. Folkbiology is the interdisciplinary perspective on how people ordinarily understand the biological world (Medin and Atran 1999). In The Native Mind and the Cultural Construction of Nature, we appreciate how far the implications of this field can go. In this overview, I choose a geographical approach: we fly from Guatemala, to Wisconsin, then to the Middle East. We then land in university classes and end up in school classrooms. This tour gives me a minimum basis for a tentative critical summary of the book. In the tour, I include not only findings but also research methods that I hope will be stimulating for readers of this journal.

\section{Guatemala: A "Common Garden Experiment"}

In a municipality in El Petén, Guatemala, three cultural groups live mostly by agriculture, hunting, and extracting

\section{E. Serrelli $(\bowtie)$}

Department of Human Sciences, University of Milano Bicocca,

Piazza dell'Ateneo Nuovo 1,

20126 Milan, Italy

e-mail: emanuele.serrelli@unimib.it

e-mail: emanuele.serrelli@epistemologia.eu forest products for sale. What happens when a land, lacking overall institutional regulation, is shared by three cultural groups with different histories? Traditional models in decision and game theory predict a "tragedy of the commons," i.e. an initial, plausibly cooperative situation soon subverted by individuals belonging to one of the groups. These individuals will begin to overexploit common resources, triggering a cascade of people switching to non-cooperative behaviors. The result will be tragic: the rapid depletion of common-pool resources. Remarkably, the same models predict that common resources will instead be preserved if - for whatever reason-all individuals of all groups maintain a resource-saving behavior. But the point is that saving up or even protecting resources is not at all a rational choice when you know that some or many others are not doing the same (or when you have good reasons to suspect so). The three groups living in Petén-native Itza' Maya, Spanish-speaking Ladinos, and immigrant Q'eqchi' Maya - seem to defy such theoretical predictions: they exhibit different patterns of common-pool resources use. Atran, Medin and their group wanted to study this intercultural scenario, exploring in particular the relation between patterns of use of the forest and ways of understanding it. In Chapter 7, "Folkecology and the Spirit of the Commons," we find an interesting description of protocols and results of this research.

A series of probes, for example, was used to reveal how people understand the forest ecology. Each informant was shown pictures of local plants and animals and was asked questions about their relations. For each plant, all animal pictures were laid out and "the informant was asked if any of the animals 'search for,' 'go with,' or 'are companion of' the target plant, and whether the plant helped or hurt the animal" (p. 185). The same was done for each animal 
paired with all plants. The pattern of relations emerging from the task is a model of the forest, a folkecological model. People are themselves placed in the models through appropriate questions: each informant is asked to "explain whether people in their community actually help or hurt each item on the plant list, and vice versa."

This experiment was preceded and followed by careful procedures. Species to be represented in the trials are not chosen at random, neither are the informats, and suitable statistical methods are used to analyze results and check for the possible (not pre-supposed) presence of cultural models that group together individual performances. I will comment further below and not go into details here, but Atran and Medin carefully explain all these passages.

Folkecological models can be analyzed in various ways. They may differ by relational richness and show prevalences and patterns. Medin and Atran found, for instance, that Itza' and Ladino groups show a similar pattern of ecological relations, while Q'eqchi' models are poorer; they also noted a prevalence of interactions involving "plants helping animals by providing them food" (p. 186). Deepening the analysis, researchers found that the Itza' have the most thorough comprehension of animals' impact on plants, including "a more nuanced appreciation of the relationship between seed properties and processing" (p. 187). Other key features of folkecological models are the prevalence of positive vs. negative relations and the appreciation and understanding of human impact on the forest, taking in some cases the form of "commitment to protecting" some species.

Atran and Medin's research shows that Itza', with their rich folkecological models, "believe (on average) that they have a lot of beneficial impact on important species" (p. 194). Itza' are the population with the longest history of living with the forest in Petén. Their traditions and language have somehow survived the seventeenth century's conquest and its consequences (pp. 165-172). Today's surviving forest, in turn, results from centuries of interactions with Itza' people. The study reveals Itza' protective attitudes that appear to contradict the "tragedy of the commons" model: "given that other people (Ladinos, Q'eqchi') do not protect the trees that Itza' do, Itza' conservation behavior does not seem to make sense from the individual's standpoint" (ibid). By understanding Itza' folkbiological cognition, Atran and Medin aim to modify the "commons" model to take account of the nontragedy situation in Petén and to draw implications for agent-based modeling of resource management.

For Itza', the forest is a player in the game, and a cooperative one. This is the consequence that Atran and Medin draw for rational-decision and game-theory accounts that otherwise model natural resources as inert objects. Folkbiological research indeed explores the "theory of mind" that Itza' at different ages apply to people and to various kinds of spirits: in brief, in managing the forest, Itza' are "playing a game with spirits" who actively protect the forest, and this could explain why they show cooperative behavior even in the absence of institutions and regulations, although Medin and Atran warn: "One should be very cautious in moving from correlations to cause" (p. 200). With studies in other spots of the world, they try to check for the reliability and generalizability of their findings, exploring the interaction among distinct factors like spiritual values, persistent contact with local habitat as opposed to migration, cross-generational transmission, and direct exposure of younger generations to the local ecology. In general, it seems, "sacred values, per se, are not enough for sustainability. At the very least, a combination of rich ecological models and sacred values may be required" (p. 205). In Atran and Medin's methodology, individual cognition-folkecological and folkbiological models in particular - is tightly linked to large-scale issues of sustainability and resource use and a "unified approach to culture and cognition can inform - and indeed transform - models of cultural cognition" (p. 208).

\section{The Distributional View of Culture and the Folkbiological Module}

Chapters 6 and 8 are devoted to the explanation of Atran and Medin's idea of culture and to the related methods of "cultural epidemiology." I start from the latter to get to the former. Once folkbiological probes and questions are set up, ${ }^{1}$ how should the informants be selected? The guideline is to maximize coverage of a group in terms of both diversity and social distance. Borrowing methods from social network analysis, researchers pick out in the group, say, six men and six women who are unrelated by kinskip or marriage, asking them to name the seven most important people of their life outside the household. Later, these first informants are asked to name, in order of priority, the seven people "to whom you would turn if there were something that you did not understand and wanted to find about the forest/fishing/hunting" (p. 209). The so-constructed social network and expert network are then extended by the "snowball method," i.e., by assigning the same task to people at their extremes. Network topology can then be studied: some networks can be diffuse, others more clustered (with clear separation in subgroups, such as

\footnotetext{
${ }^{1}$ Actually, Atran and Medin explain the involvement of people already in the phase of "tailoring" instructions and tests for the particular group (p. 57). Also, they make explicit their logic in choosing cultural groups for meaningful comparisons (Sections 3.3 and 3.4 , pp. 53-55).
} 
gender division), they can have different degrees of density, and some individuals can be "hyperconnected" (see Fig. 1).

Besides telling researchers who to administer tests and probes to, these networks are the basis for understanding the flow of information about the natural world: the assumption is that there are pathways along which the community stores, assimilates, and distributes knowledge, "pathways of learning about the forest" (p. 213). A limitation of this approach is its insensitivity to individual direct learning from experience. For Medin and Atran, however, it is likely that even "walking alone in the forest" could be driven by cultural information. Also, the authors have a peculiar theory of folkbiological learning that I will sketch out below.

A strength of network analysis is that it allows description of relational dynamics among and across groups. Researchers get evidence, for example, that Ladinos could be "at least partly parasitic" on the Itza' network (p. 214) since some nodes of the two networks are actually shared individuals, and Ladinos tend to consider Itza' people among their experts.
The analysis can thus reveal and explain the tendency toward similarity among groups.

How does knowledge circulate along the network is, for Atran and Medin, an open field of research (pp. 215-219). Social learning involves, for them, inferential processes: Ladinos, for instance, generalize from Itza' behaviors by relying on Ladino's own taxonomic and ecological knowledge (although a deeper learning is expected through contact lasting for multiple generations, p. 219). Thus, on the one hand we have a view of cultures as distributors of ideas circulating along social networks (and studied through cultural epidemiology); on the other hand, the acquisition of these ideas relies on people's cognitive processes and is constrained by them.

About folkbiological learning, I think that Atran and Medin's position can be synthesized as follows:

We suggest that much of the cultural transmission and stabilization of ideas...involves the communication of poor, fragmentary, and elliptical bits of information
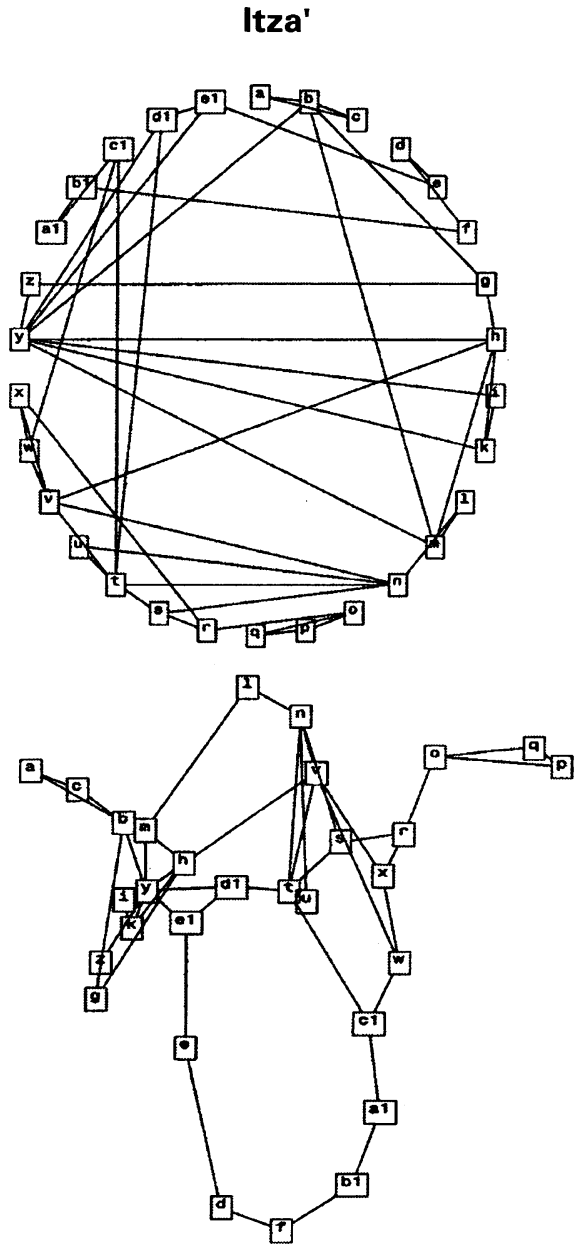

Ladinos
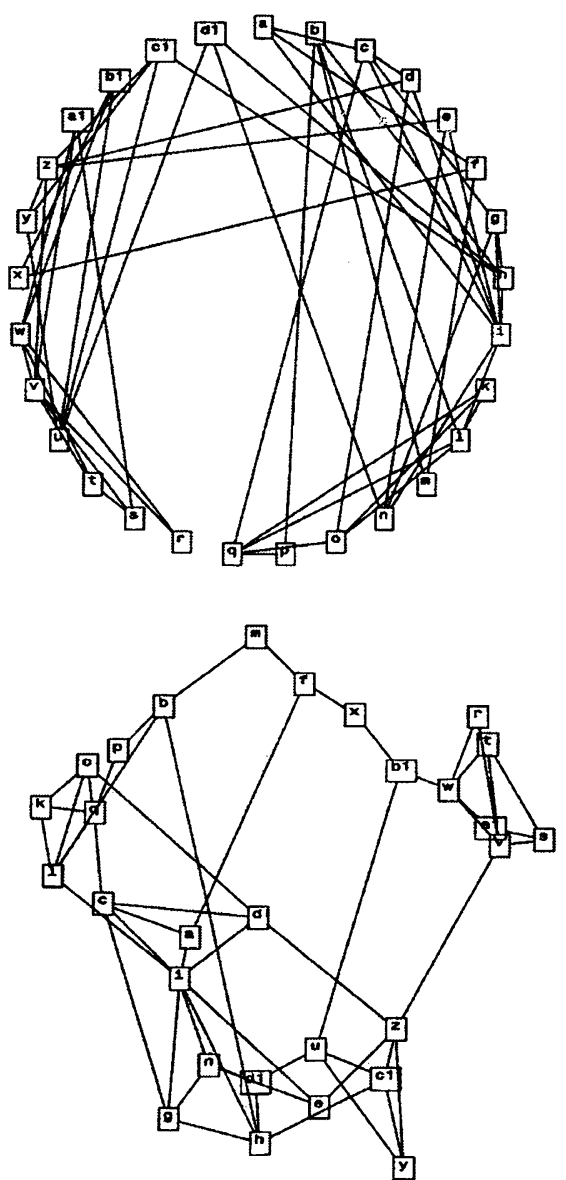

Q'eqchi'
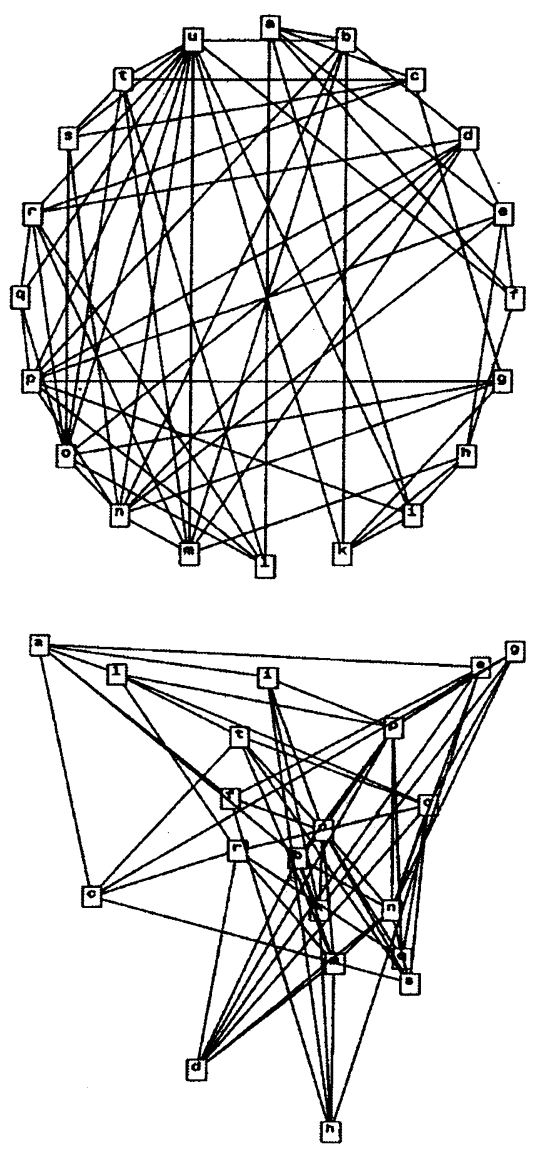

Fig. 1 Social networks fot Itza', Ladinos, and Q'eqchi' in El Petén, Guatemala. Note the different topologies describing-according to the "cultural epidemiology" approach-the flow of information about the natural world. Part of Fig. 8.1, p. 211 
that manage to trigger rich and prior inferential structures (p. 158).

Indeed, the two authors argue for the existence of "evolved cognitive attitudes" (p.223) that constrain and make possible biological theories and refer to these attitudes as a "folkbiological module." In Chapter 3, they state clearly:

There are strong, universal constraints on how people organize their local knowledge of biological kinds. These evolutionary constraints form a "learning landscape" that shapes the way inferences are generalized from particular instances or experiences. In some cases it produces consensus even though specific inputs vary widely in richness and content (p. 61).

Cultural variation is thus constrained by the folkbiological module in our minds. The latter allows us to learn biological notions through incomplete information, while it channels and limits the range of cultural differences in biological notions. To the criticism of "modularity extremism," Atran and Medin will answer in Chapter 10:

All we claim is that there is enough there, so that with some minimal interaction with the world, a number of candidates for universal principles emerge...(p. 255).

Some of these "universal folkbiological principles" we will see below, when we arrive in U.S. classrooms.

Chapter 6 differentiates the distributional view from seven alternative views. Among these, Atran and Medin recognize most affinity with "situated cognition" (e.g., Cole 1996) and "agent-based modeling" (e.g., Epstein 1999). A peculiarity of Atran and Medin's contrasting with other "distributionalists" (Boyd and Richerson 1985) is their getting rid of the expression "cultural evolution" (this is a personal comment of mine, and I will return to it in the summary). Studies of cultural epidemiology start with gathering information from individuals, choosing them in a way that allows the best appreciation of variation. Cultural variation is seen as the key to the dynamic study of change and stability. It can reveal, for example, that Itza' and Ladinos are beginning to merge. Atran and Medin point out that cultural epidemiology does not presuppose cultural groups: statistical tools like the cultural consensus model (CCM, described in Section 3.5, pp. 57-60; Romney et al. 1986) are used for analyzing patterns of agreement and disagreement. In fact, applying CCM to Atran and Medin's data often results in identifying just the acknowledged groups, but in principle, they claim to be avoiding circularity, and in effect, they are able to highlight interesting intergroup and withingroup phenomena.

\section{Wisconsin: Intergroup Perceptions of Folkbiology}

Following Atran and Medin, we now fly to Wisconsin. In their words:

Our search for U.S. biological experts led us to Wisconsin, where our attention was quickly captured by a group that has practiced sustainable forestry for more than 150 years, the Menominee. Surprisingly, instead of being seen as natural resource paragons, Menominee and other Native Americans in Wisconsin are perceived by many majority-culture hunters and fishermen as depleting fish and game resources....We decided to explore the possibility that differences in mental models of nature were a key factor in this intergroup conflict (p. 226).

In Chapter 9, "Mental Models and Intergroup Conflict in North America," Medin and Atran tell with remarkable detail (pp. 225-233) the long history of the Native American Menominee people, the many threats that were brought to their contact with land, and their "impressive resilience" to such threats (p. 233): "the Menominee," the authors write, "have demonstrated time after time that they are unwilling to trade their forest, lakes, and rivers" (p. 234). The Menominee have lived in their current reservation in Wisconsin since 1854, and they hold rights of selfregulation on hunting, fishing, and water and forest products management. Shawano County, Wisconsin, is the "majority-culture" context where the reservation is located. Despite generally good relationships and interchange, natural resources seem to be a matter of conflict: "there is resentment on the part of majority-culture sportsmen of tribes setting their own hunting and fishing regulations.... Typically these differences are seen as resource depleting" (p. 236). Quite evidently, this is an ideal situation where Atran and Medin can study folk models.

A fundamental element of their approach I didn't mention before is ground-truthing:

The maintenance of the forest in light of large-scale deforestation in the surroundings as well as constant economic incentives to the contrary [is] quite impressive and provide a literal ground-truthing of Menominee respect for nature (p. 234).

The study of mental models is incomplete without corresponding observations and measurements of actual practices, all the more so if researchers aim - like Atran and Medin - to convince other scientists and to influence policymakers (see, e.g., "Conclusions," p. 269). Also in the case of Petén, self-reporting of forestry practice was not at all sufficient to establish differences among the three groups: researchers measured plot sizes, species diversity in plots, number, coverage and frequency of trees, soil 
chemical composition, dimension and dispersion of fires, and so on. Mental models correlate with patterns of use, which can be observed, and the effects measured when you "map different patterns of use onto an overall measure of destructiveness" (p. 174).

Getting back to Wisconsin, Atran and Medin point out the health of Menominee forest in comparison with surrounding deforestation, a fact that one can appreciate with a simple look at satellite images (try it). Observations of this kind, together with the fact that Menominee have carried on self-regulated forestry practices for 150 years at least, are the necessary ground-truthing of studies of mental models and intra- and intergroup perception.

A naive view would oppose the close-to-nature, deeprooted and forest-committed Menominee to the shallowrooted, ignorant and resource-depleting majority-culture sportsmen. Atran and Medin's study give us a much more complex view: the mental models and amount of ecological knowledge appear pretty similar in the two groups. Rather, subtle differences in the organization or "orientation" of their knowledge would be the source of reciprocal misrepresentations and stereotypes, reinforced by mass media coverage. Several probes were administered to experts from the two groups about fish and fishing. They exhibited consensus on species categorization and showed detailed knowledge about the life cycle and ecological relations of fish. According to Medin and Atran, however, knowledge in the two groups is differently oriented: "majority-culture experts organize their knowledge around goals that target adult fish" (p. 239) and are less ready to give answers in terms of life cycle and ecology. Group differences disappear if tasks are run at a slower pace. Another difference between the groups is the degree of robustness of folkbiological models across the distinction between experts and non-experts, which in the Menominee is higher.

I think that many readers will enjoy how Atran and Medin's group sets up probes and tests to explore the aspects of folkbiological knowledge which are more relevant to their research questions. In the case of Menominee and majority-culture in Wisconsin, researchers "wondered how the differences in orientation might be reflected in values and attitudes toward different fishing practices" (p. 240) and therefore set up three tasks: (1) ranking of fish, to assess the relative importance of different species for the individual; (2) ranking of goals, to ground the idea that groups go fishing with different aims; (3) rating of fishing practices, wherein the authors expected to see reflected the values concerning fishing. Having found broad intergroup commonalities and similarities in all three tasks, they had the idea of asking informants of each group for detailed expectations upon the answers by people from the other group, as well as by the average person from his/ her own group. With this procedure Atran, Medin and colleagues were able to show and analyze misperceptions and perceptual amplification of intergroup differences: "majority-culture fishermen hold strong, incorrect expectations concerning Menominee attitudes and values" (p. 248). In reading these reports, I grasp very clearly how encounters between different "folkbiologies" can have dramatic effects on evironmental policy and decisionmaking at all scales, even - and this is the case-when line common values and interest in conservation are present. These very analyses of misperceptions and search for their sources (pp. 249-253) are, for Atran and Medin, a necessary contribution.

\section{Middle East: The Frontier of Reseach}

From the forest of Wisconsin, before ending in our classrooms, we fly to the West Bank and Gaza. Although the book is very brief about these scenarios, I think it is meaningful to see The Native Mind and the Cultural Construction of Nature as a stepping-stone in an ongoing, expansive research program. Atran, Medin and their group involved themselves in new field sites in the Middle East, Southeast Asia, and elsewhere: quite recent papers report surveys of Jewish Israelis living in the West Bank and Gaza (Ginges et al. 2007), studies with Palestinian and Israeli leaders (Atran et al. 2007), and others about "emerging clashes between the Muslim and Judeo-Christian world" (p. 272). Such an iterated "branching out" of research is fueled, I think, by several factors. One can be the need for more varied empirical cases for the generalization of models and specific findings. But another factor is the aspiration that the cognitive-anthropological analysis developed for Petén and Wisconsin be somehow applied in all cases of cultural and political conflicts, being of some help in the search for solutions. In this movement, I see Atran and Medin making it clear that folkbiology and folkecology are likely to be at the very heart of the great majority of conflicts. The centrality of environmental resources for life is related to the ways people think about resources and act upon them. Spiritual values involving nature and land can have dramatic effects on cognitive handling of conflicts. By proposing "rational" trade-offs, for example, politicians can yield effects which are contrary to their goal: in political disputes like the Israeli-Palestinian conflict, instrumentally driven trade-offs can exacerbate violent opposition to compromise solutions, "while noninstrumental symbolic compromises may reduce support for violence" (p. 272).

Research is stemming out of contexts that are theoretically and socially uneasy. In reporting results, this implies an uncommon amount of effort in justification and response to critics, as is evident throughout this book. I take as an example 
pp. 255-258 in the "Conclusions," where Medin and Atran briefly (once again) respond, in order to critics of modularity and charges of biological determinism and to critics of the analogy between cultural and biological evolution, to the possible bias of their research in favor of neglected and oppressed groups and to the charge of shallow ethnography. I will return to some of these criticisms in my summary, but I think that part of these researchers' exposure is related to their involvement in situations of conflict, of possible cultural misperceptions, of unequal distribution of resources of endangered societies and cultures; and it is also due to their strong commitment to their studies, that they believe potentially relevant to policy making and problem solving. This kind of research requires adopting a multidisciplinary approach, sometimes perhaps stretching the boundaries of disciplines a little bit more than what is normally allowed, and it is also demanding in economic terms:

Detailed analyses of the relations between ecology, technology, social networks, and so forth require large interdisciplinary efforts, over many field seasons, at a cost that usually exceeds typical ethnographic fieldwork by one or several levels of magnitude (p. 161).

It is evident that for this group, the game is worth the candle.

\section{U.S. Undergraduates: A Degenerated Population}

We arrive now at the most used research context for cognitive psychology: university classes. Bringing together their folkbiological studies in small-scale societies, Medin and Atran substantiate the (indeed, familiar) criticism of generalizations drawn from the undergraduate population. They formulate the "cautionary tale" that "at least in the case of folkbiology, standard populations may be nonstandard and vice versa" (p. 114), and cognitive processes considered as normal might be actually the "failure to undergo 'normal' perceptual development" (p. 110). In Medin and Atran's account, we humans have a mental module: the FBS, folkbiological system (they argue at length about conditions, distinctions, and counterarguments for this thesis, e.g., on pp. 63-67 and 114-119). The module is innate and universal, but it needs contact with nature for its development. Undergraduate students (and non-expert Western people in general) lack such necessary contact, and their atypicality becomes clear in comparison with other cultural groups: from "standard population" they become the "odd group out."

Chapter 4 is the longest (almost 60 pp.) and most difficult of the book, and it is itself a description and tentative synthesis of several other works. So my synthesis here will be very rough, just an invitation to read directly to appreciate the complexity of the research and objectives. The central core of the chapter is people's biological induction, i.e., their use of reasoning for a generalization of biological properties from a taxon to more inclusive groups. The other pillar of the chapter is folktaxonomy, i.e., the hierarchical organization of taxa which is the necessary support for biological induction. I focus mainly on induction and leave taxonomy for the next section.

The study of undergraduates (Osherson et al. 1990) has elucidated how they use a model called SCM (similarity coverage model) in which biological induction relies on perceived similarity. Students' induction was tested by means of questions such as: "if species $\mathrm{X}$ and $\mathrm{Y}$ are subject to a disease, how likely is it that all $\mathrm{Z}$ will be subject to such disease?" $\mathrm{X}$ and $\mathrm{Y}$ could be, for example, cow and mouse, and $\mathrm{Z}$, mammals. It was shown that similarity was the base for induction: indeed, inductive strength (i.e., willingness to generalize) was increased if $\mathrm{X}$ and $\mathrm{Y}$ were typical organisms, similar to the majority of the category (typicality effect); strength of induction was also increased if $\mathrm{X}$ and $\mathrm{Y}$ were two highly diverse organisms (diversity effect). In both cases, a good coverage is based on similarity and justifies induction. In Chapter 4, Medin and Atran put into discussion this model and strongly criticize its supposed definition as a "standard" or "basic" model of biological reasoning.

Diversity effect, for example, is completely absent in Itza' Maya and U.S. experts, whereas undergraduate students appear to learn it and to increasingly use it throughout the tests (p. 87). Interestingly, diversity effect was also observed to a certain degree in some U.S. taxonomists (p. 73): Medin and Atran then consider the idea that scientific systematics might be one factor influencing biological reasoning. Diversity effect could be explained by a learned theory of context-free, "causal unity underlying disparate species" (p. 72).

But the most important point here is that Itza' and experts use a completely different kind of reasoning for biological induction: they preferentially use causal, contextsensitive, ecological connections as a guide for induction. Simply put, for example, a disease will be spread among species if they entertain ecological relations, if their geographical ranges overlap, and so on. Quite interestingly, Medin and Atran found evidence that undergraduates do not lack some degree of ecological information. Rather, they do not consider it relevant (Sperber and Wilson 1986) to biological inference. Students normally rely heavily on abstract reasoning, but they can purposefully be helped to bring background knowledge that violates similarity into relevance (see pp. 91-92): with "careful attention...reasoning strategies that are salient among experts and Itza" can be brought out in students (p. 92). 
Comparative studies also put typicality effect into discussion. Undergraduates make strong use of the central tendency criterion: a typical organism is one that is perceptually average in the category. Accordingly, typicality seems to be determined by the intrinsic structure of the biological domain. But in all other groups - and indeed in deeper studies of the "standard" population-typicality appears to depend on extrinsic factors, like desirability, familiarity, and idealness. Once again, the standard population is revealed to be the odd group. Atran and Medin draw the need for an "alternative approach" to biological reasoning, one that "allows for strong influences of more extrinsic factors like ideals, goals, and habits of mindeven in domains that, like biology, have rich instrinsic structure" (p. 100). Many readers will likely be interested in consequences for learning, which Atran and Medin make explicit:

We think that it is very unlikely that category learning is a passive, bottom-up process that reflects intrinsic structure without bias. It is much more likely that the most relevant (and ideal) examples are learned first and that these initial representations guide further learning (ibid).

Summing up, the folkbiological cognitive system of people populating major universities is underdeveloped due to the lack of contact with the natural world. Nonetheless, models of biological cognition have been built on such "standard" population: taxonomical relations based on perceptual similarity have been seen as the support for biological induction, guided by principles of typicality and diversity. Similarity has been considered an intrinsic structural property of the biological world, detected by perception. Alternative models are required to account for biological inference in almost all other groups, such as small-scale society peoples and U.S. experts. For these groups, ecological and causal relations prevail on taxonomy in induction; the diversity effect is not supported by an abstract and generalized principle of "common cause," and typicality is strongly influenced by cultural factors. These new models can also describe biological cognition in undergraduates, furthermore explaining its "devolution" and offering some guide for teaching biology.

\section{In the Classroom: Urban U.S. Children}

Finally (actually, in Chapter 5 out of ten), we see researchers interviewing children in classrooms, looking at textbooks and analyzing practices in science education (diagrams, discourse style, compatibility between school and community, etc.). Their findings in this field are only at a preliminary stage, but the whole research program seems to bear a message for education, i.e., the critical importance of understanding the relationship between formal and informal learning and the fact that "cultural knowledge, values, and models are an important part of the story" (p. 141). Science education is not culturally neutral: it can conflict with folkbiology on the level of facts, in how knowledge is organized, as well as in cultural models and values. A particularly telling example cited by Medin and Atran is the following:

...Menominee children have a precocious understanding of biology. Indeed, on standardized tests, fourthgrade Menominee score above the national average in science and it is their best subject. Strikingly, however, by eighth grade, science is their very worst subject and they score below the national average. The fact that reading scores do not show a corresponding drop suggests that something peculiar to science instruction is the key. At a minimum these observations indicate that the educational systems are not taking advantage of the knowledge that Menominee children are bringing to the classroom (p. 138).

Even the youngest Menominee children show patterns of induction based on ecological relations. They don't show a pattern which has been long studied in urban U.S. children, i.e., anthropocentric asymmetry of induction: urban children are more willing to generalize from humans to animals than vice versa, and this asymmetry was seen as evidence that folkbiology derives from folkpsychology (Carey 1985). Atran and Medin, supporting the idea of an independent folkbiological module, challenge this view: the anthropocentrism observed in young children "does not reflect a failure to distinguish biology from psychology. Part of the story may be that humans are the only biological entity that urban children knew very much about" (p. 122). Research with Maya, Menominee, and rural children brings evidence that familiarity is an important factor in induction. Children's familiarity with many species and with ecological relations makes the anthropocentric asymmetry disappear.

U.S. children, and above all those who live in an urban environment, are for Medin and Atran under the effect of devolution. In Chapter 2, "Universals and Devolution," they explain the concept and give some ground-truthing to it by statistically analyzing the names of trees in the Oxford English Dictionary from 1525 to 1925 . As usual, the analysis is not simplistic and tries to explain correlations and tendencies in detail. In the twentieth century, terms for naming trees undergo a steep decline. Linguistic richness is correlated with richness of experience, but extinction of terms can be slower than the "extinction of experience" (i.e. devolution): "the language may preserve certain 
distinctions beyond the time these distinctions are still understood....When knowledge declines, the structure, like an abandoned building, may remain for a while" (p. 47). This lapidary expression bears the serious view Medin and Atran have of the situation in highly industrialized societies and the related cognitive effects with which scientific instruction must deal every day.

Shrinking of terminology is not all. There is also a decrease of specificity, and this relates to the important theme of folktaxonomy. We consider it briefly now. Medin and Atran make a strong and clear claim on taxonomy:

Biological taxonomies seem to be culturally universal; and they are structured enough to impose constraints on possible theories (p. 28).

Broad, cross-cultural similarities in folktaxonomy-and between folktaxonomy and scientific systematics-are for Atran and Medin once again evidence of the existence of a mental module that, suitably stimulated, organizes the biological domain. The nested categories of folktaxonomy are: kingdoms, life forms, generic species. Species are often subdivided into folkspecifics, where the correspondence between folk and scientific systematics begins to blur. Intermediate folktaxa can also be possible between generic species and life form. Folk kingdoms distinguish plants and animals from the rest of the world:

From an early age, it appears, humans cannot help but conceive of any object they see in the world as either being or not being an animal, and there is evidence for an early distinction between plants and nonliving things (p. 29).

Life forms (e.g., "tree") are more specific, characterized by a small number of perceptual diagnostics. The most universal and conceptually important category is generic species:

Ethnobiologists...tend to agree that this level best captures discontinuities in nature and provides the fundamental constituents in all systems of folkbiological categorization, reasoning, and use (p. 31).

Children share all the universal aspects of folkbiology. They have, for example, the idea of an "underlying causal nature, or essence" (p. 21), tested through probes on heredity and questions on fictional cross-species adoptions (section 5.3 of the book). Their folktaxonomical capacities are in place despite the steep devolution and loss of familiarity with nature they are experiencing, and "it is little wonder" for Medin and Atran since

Folkbiological taxonomies tend to be among the most stable, widely distributed, and conservative cognitive structures in any culture. Once set into place, such a structure would likely survive even catastrophic historical unpheaval to a clearly recognizable degree (p. 36).

Similar taxonomies can be used differently: undergraduates use taxonomy as a "stand-in for ideas about the likely distribution of biologically related properties," whereas "for the Itza' (and other knowledgeable groups), the taxonomy may constrain the likely operational range of ecological agents and causes" (p. 114). Categories of folktaxonomy can exhibit different relative salience and inductive force: Itza' can be unwilling to generalize above the species level, whereas undergraduates and urban children can be willing to generalize to life form level (loss of specificity). If you want more information, you must read Chapters 4 and 5 of the book: "Devolution and Relative Expertise" and "Development of Folkbiological Cognition."

Still there is-for Atran and Medin-room to help children improve their biological reasoning and to try to set up science instruction in a way that respects folkbiological cognition of so-called "minority groups"-appearing, in this case, a model for the socalled "standard population." And here I close with a suggestive quotation on the topic:

Imagine tourists from a country where people drive on the right side of the road are transported to another culture where people drive on the left. [As pedestrians], all of their attentional habits for looking before crossing will only get in the way.... The analogy is straightforward: the set of practices Native American children bring to the classrooms are ones that are natural outside of school, but create a clash in the classroom, because the rules are different there (p. 274).

\section{Critical Summary}

The Native Mind and the Cultural Construction of Nature is a valuable and rich product of an outstanding research program that is growing in far-off spots of the world, brought forth by a multidisciplinary, lively community of experts with their graduate students and driven by fascinating questions like: How do people conceptualize nature, how do they organize taxonomy and induction in the biological domain? How does understanding of nature relate to decisions and actions? How is individual cognition related to culture, and how does it vary across and within cultures? How is cognition affected by a prolonged lack of contact with natural environment? How is culture transmit- 
ted and developed along social networks? Can a focus on biological cognition make sense of intergroup conflicts and help to find ways out of them?

This book is not a manual, definitely. Rather, it is a super-rich work-in-progress of what is now becoming a research tradition at the intersection of different disciplines. Such essence makes the book very demanding, but very attractive too. Believe it or not, there are many, many more questions and tentative answers therein than those I listed above. In reading, I had the exciting - and, at times, frustrating - feeling of being thrown into the ongoing research: I followed questions germinating one from the other; I attended to the smart design of experiments and to the struggle to interpret a large amount of data, getting into them from several, slightly different points of view; I assisted with the diligent filling-in of background information (historical, theoretical), and so on.

One thing I personally like in the book is the complete absence of the binomial expression "cultural evolution." It is not that the authors fail to recognize the analogies between their distributional view of culture and evolutionary biology: they remark in theoretical paragraphs that "like modern biology, the distributional view of cultural phenomena does not take individual variation as deviation but as a core object of study" (p. 221); they notice that in both domains, variation is seen as the basis of dynamics. Studies of cultural change that have some analogy with biological evolution are often put under the rubric of "cultural evolution." In this case - as well as in others, I believethe label could be particularly confusing: biology pervades the book as the content of cultural knowledge and cognition, and evolutionary biology is also called forth as a framework - if not an explanation - for the existence of a folkbiological module in our minds. Invoking evolution to also support-like a blueprint - the models of cultural change would have been really misleading. Perhaps Medin and Atran's example could promote the simple and sufficient expression of "cultural change."

Among possible criticisms of this work, I select two: one on the modularity of mind and one on what the authors call "scientific biological taxonomy." I will close then with my most important criticism, about the book's structure.

Many authors have pointed out epistemological flaws and empirical problems arising in evolutionary explanations of this or that particular characteristic of the human mind. "The Spandrels of San Marco and the Panglossian Paradigm: A Critique of the Adaptationist Programme" [Gould and Lewontin 1979] is an early and paradigmatic example of such reaction; The Growth of Biological Thought. Diversity, Evolution, and Inheritance [Dupré 2003] is more recent and specific on evolutionary psychology). I must say that Atran and Medin show that they are aware of the traps of backwards extrapolation and construction of adaptive "just- so stories" for the structures we are interested in legitimizing (e.g., pp. 63-65, 114-118, 255). Indeed, they emphasize evidence for this module existing and operating here and now, and this seems to reduce the importance of evolutionary support. Even so, the many modulations described, and the formalization of the concept of "devolution"-namely, the importance of natural and cultural environments in cognitive development - seem to leave us with weak support for the existence, uniqueness, autonomy, and importance of the folkbiological module. Much weaker, indeed, than the support we have for a linguistic module (see Sterelny 2003). Too weak, maybe: the existence of the folkbiological module looks like an unfalsifiable assumption, defended by means of a growing number of ad hoc hypotheses that explain its variation across cultures. In this light, I think that Atran and Medin's arguments are best seen as the defense of a working hypothesis that is proving to be useful in their program. Readers and researchers should not forget, however, that such a working hypothesis rules out mechanisms of social learning that - for other authors - could be largely sufficient, in conjuction with the structure of the natural world, for an elegant explanation of folkbiological universals and variations among different cultures (e.g., Sterelny 2003, Chapter 10).

About scientific taxonomy, I hope that at a certain point of their research this group engages more thoroughly the lively debate on taxonomy and systematics which is going on within science and philosophy of biology (e.g., Mayr 1982; Ereshefsky 2001). By now, Atran and Medin treat scientific taxonomy as a system which is external to the analysis, a system that folktaxonomies can approach more or less closely. By doing so, although they present scientific taxonomy as an historical product (pp. 25-28), they tend to consider it as a rather unitary, established ending point. This move perhaps plays a role in their argument for folkbiology, but it neglects, for example, the existence of different scientific taxonomical schools (see, e.g., Hull 1970; Haber 2008), of different views that sometimes compete and conflict in the endeavor of ordering the biological world. Symptoms of some of these dynamics are reflected in Atran and Medin's own exposition where they put forth scientific taxonomy at times as phylogenetic organization, while other times as based on similarity. They are definitely aware of the debate (e.g., p. 277), and their choice is deliberate (e.g., p. 77). Ecological reasoning, emphasized in the people of small-scale societies, is a further part-a struggling part sometimes - of scientific taxonomy. Cognitive variety and complexity is thus seen in science as well. Probably, this will be a relevant and necessary topic for the deepening of the research on biological reasoning. Surely, it is relevant for biology education (e.g., Thanukos 2009). 
The aspect of this book I want to criticize most seriously is its organization. I already mentioned the vastness and complexity of content. In short, I do not think that the book's structure helps the reader to approach it and become involved. Chapters 1 to 3 (pp. 1-61) seem to me a long, long introduction collecting disciplinary justifications, theoretical definitions, broad general claims, a brief history of taxonomy, a survey supporting devolution - a concept that in my opinion only becomes clear in Chapter 4-and other sub-arguments. Folk examples in this part of the book are poorly contextualized since they concern populations that will be introduced later in the book. On the other hand, the "Material and Methods" section (Chapter 3) is redundant with other parts (e.g., Chapters 7 and 9). With Chapter 4, things don't seem to get better: this is an astonishingly long chapter (60 pp.), dedicated to the complex task of bringing together evidence on a range of topics and from a number of different research projects. While all the reported findings are interesting and important, the way they are presented is not facilitating and inviting for the first approach of a general reader. I thought that the book could instead be seen as a huge expansion of a paper, with the first part containing the (multi)disciplinary context, background, and potential competing visions addressed to colleagues (the choice to begin in Chapter 4 with undergraduate students may indeed seem a disciplinary standard in cognitive psychology). But the "long paper" vision does not hold up well since significant pieces of theoretical background are also scattered throughout the book (e.g., Chapters 6 and 8). The second half of The Native Mind and the Cultural Construction of Nature is overall more accessible, and that is why I started my review from there. I don't think that the complexity of a research program can justify an organization which is not functional for the reader, or at least for a selected kind of reader.

In sum, the structure of this book does not help our approach to this interesting and important research. The content is all in there anyway, and I hope that this review will be useful as a "tourist guide" for readers and that it may also serve in encouraging people to read the book and to become sensitive to this ongoing, remarkable research enterprise.

\section{References}

Medin D, Atran S, editors. Folkbiology. Cambridge: MIT Press; 1990.

Cole M. Cultural psychology. Cambridge: Harvard University Press; 1996.

Epstein J. Agent-based computational models and generative social science. Complexity. 1999;4:41-60.

Boyd R, Richerson P. Culture and the evolutionary process. Chicago: University of Chicago Press; 1985.

Romney AK, Batchelder W, Weller S. Culture as consensus: A theory of culture and informant accuracy. Am Anthropol. 1986;88:313-38.

Ginges J, Atran S, Medin D, Shikaki K. Sacred bounds on rational resolution of violent political conflict. Proc Natl Acad Sci. 2007; 104:7357-60.

Atran S, Axelrod R, Davis R. Sacred barriers to conflict resolution. Science. 2007;317:1039-40.

Osherson D, Smith E, Wilkie O, López A, Shafir E. Category-based induction. Psychol Rev. 1990;97:85-100.

Sperber D, Wilson D. Relevance: communication and cognition. Oxford: Blackwell; 1986.

Carey S. Conceptual change in childhood. Cambridge: Bradford Books; 1985.

Gould SJ, Lewontin RC. The spandrels of San Marco and the Panglossian paradigm: a critique of the adaptationist programme. Proc R Soc Lond B. 1979;205(1161):581-98.

Dupré J. Human nature and the limits of science. Oxford: Clarendon; 2003.

Sterelny K. Thought in a hostile world. The evolution of human cognition. Oxford: Blackwell; 2003.

Mayr E. The growth of biological thought. Diversity, evolution, and inheritance. Cambridge: Belknap; 1982.

Ereshefsky M. The poverty of the Linnaean hierarchy: a philosophical study of biological taxonomy. New York: Cambridge University Press; 2001.

Hull D. Contemporary systematic philosophies. Annu Rev Ecol Syst. 1970;1(1):19-54.

Haber MH (2008) Phylogenetic inference. In A companion to the philosophy of history and historiography. Oxford: Blackwell Publishing.p. 231-242.

Thanukos A. A name by any other tree. Evo Edu Outreach. 2009;2 (2):303-9. doi:10.1007/s12052-009-0122-7. 